\title{
The Research of Restraint Design Theory Based on the Evolution of Product Technology Systems
}

\author{
Bing Lv \\ Mechanical Engineering College \\ University of Jinan \\ Jinan, Shandong Province, China \\ me1vb@ujn.edu.cn
}

\author{
ChangQing Gao \\ Mechanical Engineering College \\ University of Jinan \\ Jinan, Shandong Province, China \\ me_gaocq@ujn.edu.cn
}

\begin{abstract}
Technology system has been in evolution. Solving contradictions of technical system is the driving force of evolution. All technical systems evolution follows certain objective laws. As an organism, the technical system itself is constantly changing. When the technology innovation evolutionary path through certain areas of the system, Lead to serious loss of human society and the natural ecological This area is known as evolutionary path of negative trap. Of course, regional boundaries and location of negative trap can ascertain through technical system evolution forecast. In other words, In the course of technical system evolution, we could avoid the trap of negative effective by advance judgment, build an immune innovation mechanism about of the technology innovation system which improve our innovation mechanism by abstinence design, ultimately benefit human society.
\end{abstract}

Keywords-Restraint Design; Technology Evolutionary; Negative Trap

\section{INTRODUCTION}

Natural harmony is the core of the Taoism of ecological thinking, the nature of human society is a natural, natural center of the world, everything, including the highest ecological ideal of human society is completely state of the natural realm. Human vocation is to love nature, protect the environment and protecting the ecology, the pursuit of natural ecological harmony.

"Southern Journal" bimonthly from May to June, Uruguay, published articles on the global rich and the poor conditions, as well as the harm caused by the excessive consumption of health and ecological environment. Excessive consumption stimulates the pace of innovation of technology systems, causing huge waste of the world's natural and social resources. In other words, the evolutionary trajectory of continuous scientific innovation trajectory to guide mankind toward the abyss beyond redemption we must be alert and emergency adjustment of innovative mechanisms. [1]

\section{CONTEMPORARY DESIGN METHODS TO EXPLORE}

The development of contemporary science, especially biology, genetics, physics, astrophysics, and artificial intelligence breakthroughs, forcing people can not adapt to it, but cross to the deeper areas.

Humanity must learn before acting more comprehensive ability to detect the crisis. Human behavior decision-making-features designed to improve economic management, social management and planning of the future level of human existence up. Based on this, one after another emerged in many design methods.

Green design is built at the end of the 1940s, which is the continuation of the rapid development of environmental ethics and the environmental movement later in the 1960s. It is a way of thinking and the integration of production from the social macro perspective on the relationship between human activities and natural and social aspects. In actual operation, the green design impact on environmental protection positive and far-reaching. However, green design is difficult to solve the social crisis.

Slow design is a philosophy. According to this thinking, the world is the birth of a new design mode. The role of design is in harmony with the environment conditions to maintain the balance of social, cultural and individual needs. In this mode, the time is not a major constraint, do not have to respond quickly to market and compete with rivals, no longer seek to minimize or maximize, or the fastest. The purpose of the design is to ensure that the aesthetic needs of personality and social and human balance of the Earth. Slow design, the ideal design state, but does not indicate how to solve the crisis brought about by design.

Low-carbon design requirements for the design department and designers to use energy saving, low consumption, non-polluting and recyclable as the primary starting point of the design behavior, from the initial stages of product development, cope with the impact throughout the product life of nature and the environment by selecting the appropriate technology and materials. Low-carbon design concerns only in terms of carbon emissions, and little for other elements.

Through the study of a large number of patents, Altshuller found that, as an organism, the technical system itself is constantly changing.

Technology systems adapt to the environment through continuous self-adjustment. "Good" technology adapts to the changing environment through continuous self-adjustment for survival and development; because "Poor" technology systems

This work is supported by NSF of Shandong Province (ZR2010EL026) and the National Natural Science Foundation of China (No. 50905074). 
can not adapt to environmental change, extinction is the inevitable result.

\section{The RESEARCH OF RESTRAINT DESIGN THEORY}

The current prediction theories have two types of Western theory and theory of the Soviet Union. One important way in the first category theory is a mathematical model based on data from past and present technology.we predict by extrapolation of the parameters in the mathematical model changes. The second category is the TRIZ technology evolution theory. The theoretical analysis of the world's patent library, and confirmed the trend of structural evolution, technology evolution patterns and the evolutionary line.

Product technical maturity forecast refers to the product as a technical study, which predicts what stage of the current products in the technology life cycle by evaluation of the current product technology. Making sure the position of products on the S-curve is TRIZ technology evolution theory research, known as the maturity of the product technology forecast.

I found that the existing system of technical innovation and evolution path through some of the region through research, will give the human society and natural ecosystems a loss which can not be intended to complement. This region is called the negative trap.

For circumvention of technological evolution in the process of negative trap, I propose the research of restraint design theory. Reads as follows:

The idealistic goal of technical system evolution is often difficult to achieve. Because the evolutionary process will continue subject to the interference of external factors, the result of evolution cause many by-products, or called secondary products. This evolution is almost irreversible, and even can not compensate for. If the fuzzy front end of the product technology systems development will be able to predict these disastrous consequences, we can do to stop doing. By appropriate technical means to pre-judge the regional boundaries and the specific location of the negative trap, effectively avoid negative traps, and construct of innovative technology systems immune innovative mechanisms. To promote a more complete theoretical system of the invention and innovation, we can be done in harmony with nature. I named this process for the restraint design process of the evolution of technical systems.

As we all know, many of today's industries are built on top of the ruthless plundering of the natural; For example, the development of automobile industry this day, has almost reached a situation of depletion in the Earth's oil resources, and eventually destroy the Earth's ecological resources. But this industrial development is irreversible, and the negligible role of remedial and compensatory measures for this situation. Only restraint design tools could effectively avoid the consequences of catastrophic evolution.

\section{The Theoretical Basis of Restraint Design}

The evolutions of technical systems have certain rules which can be found, and are in line with nine laws. They are: increase the idealized level; non-balanced development of the subsystem; dynamic growth; pass to complex systems; pass to the micro system; reduce path length of the energy flow; autonomous system integrity; increase controllability; increase harmony. [2]

Characteristics of the evolution of technology systems reflected in: Integrity; imbalance; open; purpose; non-linear; coherence; initial state of extreme sensitivity.

The basic meaning of the non-linear: Due to the presence of nodes evolution of technical evolution process, it results in a technical system and its subsystems being in the chaotic state at the technology evolution node. The entire evolutionary process Expressed as linear segments forming to a non-linear process.

The basic meaning of the extreme sensitivity of the initial state: According to the complexity of the theory, the initial state of the system even if only subtle differences, then the subsequent behavior is completely different, this characteristic of the system is known as the "Butterfly Effect". System is characterized by a subtle change in technology nodes, which will likely lead to the formation of a huge group.

Design innovation process is actually a problem-finding, problem-asking and problem-solving process. Every innovation in the product development process, are the problem-solving process. Theory of Inventive Problem Solving, as a world classic theory, which core, is to solve conflicts phylogenetic. Idealization is an important way to solve the problem in the TRIZ theory:

$$
\text { Ideality }=\Sigma \text { Benefits } /(\Sigma \text { Expenses }+\Sigma \text { Harms })
$$

Costs include the cost of raw materials; the space occupied by the system; the energy consumption and the noise. Hazards include waste and pollution.

Through the above formula, we can understand the level of technology systems idealized by the effectiveness, costs and endanger. The benefits and costs are easy to measure obviously. But costs of harm or side effects are difficult to calculate; this consideration also changes over time, if this change is positive, we can also accept; if negative changes occurred, but not controlled, will have catastrophic consequences, and greatly reduce the level of technology systems idealized.

We know the market demand is the driving force of the development of technical systems, but also the technical system variable node. Understanding of and access to the user's needs is often the first step of an innovative, through research, we found that the demand in the evolution of the state, which evolution governed by objective laws, and 5 law of demand evolution. Evolution idealized that demand; dynamic needs evolution; demand evolution coordination; demand evolution integration; demand evolutionary specialization. In many cases, the demand evolution idealized achieve by demand evolution dynamic, demand evolution integration and demand evolution 
specialization. After 3 and often achieve by demand evolution coordination.

Needs evolution idealized refer to increasing the number of demand, improving the quality of the demand, reducing the time consumption of these needs, costs and side effects, which is the trend of the demand evolution? Qualitative description using the formula:

$$
I=\frac{\sum_{i=1, j=1}^{I} Q_{i} Q_{j}}{\sum_{k=m}^{0} C_{k}+\sum_{l=n}^{0} H_{l}}
$$

I - The demand evolution idealized level

Qi - Number of needs

$\mathrm{Qj}$ - Demand for quality

$\mathrm{Ck}$ - Meet the demand for the cost required, including time

\section{$\mathrm{Hi}$ - Demand side effects}

In the formula, the demand evolution idealized level tends to infinity is the demand evolution idealized evolutionary trends. There are four methods to promote the evolution of idealized demand this trend, which are increasing the number of demand, improving the quality of the demand, reducing the time and cost to meet demand, reducing the side effects of realized demand.

\section{SUMMARY}

So technology system evolution is the development in accordance with their own evolutionary route, During this period, and continuously driven by market demand evolution and its evolutionary trajectory occurs a certain degree of change, Such a change will undoubtedly cause some to distort the idealized process of technical systems, and ultimately produce some secondary technical system. Only through restraint design theory study, Predict means to circumvent the excessive demand caused by the evolution offset idealized; can plan for the phylogenetic best trajectory, provide a theoretical basis for countries to optimize the industrial structure. Lotus Just Buds, dragonfly stand on top of it already. Faced with the new changes in market demand, enriching the universal critical condition; restoring the influencing factors of the evolution of technology systems, and indicating the direction of evolution of product technology, are the key of restraint design process.

\section{REFERENCES}

[1] Information on http://news.sina.com.cn/s/2004-06-23

[2] Wucheng Zhang: Technology Innovation Method Overview (Science and Technology Press, China 2009). 